\title{
Lepidopterofauna (Papilionoidea e Hesperioidea) do Parque Estadual do Chandless e arredores, Acre, Brasil
}

\author{
Olaf Hermann Hendrik Mielke $e^{1,2}$, Eduardo Carneiro ${ }^{1}$ \& Mirna Martins Casagrande ${ }^{1}$ \\ ${ }^{1}$ Laboratório de Estudos de Lepidoptera Neotropical, Departamento de Zoologia, \\ Universidade Federal do Paraná - UFPR, CP 19020, CEP 81531-980, Curitiba, PR, Brasil \\ ${ }^{2}$ Autor para correspondência: Olaf Hermann Hendrik Mielke, e-mail: mielkeomhesp@ufpr.br
}

MIELKE, O.H.H., CARNEIRO, E. \& CASAGRANDE, M.M. Lepidopterofauna (Papilionoidea e Hesperioidea) of the Parque Estadual do Chandless and surroundings, Acre, Brazil. Biota Neotrop. 10(4): http://www. biotaneotropica.org.br/v10n4/en/abstract?inventory+bn03210042010.

\begin{abstract}
Given the absence of Lepidoptera inventories in the State of Acre and its scarcity in the Brazilian Amazon forest, this study aimed to list the species of Hesperioidea and Papilionoidea present in the Parque Estadual do Chandless and surroundings. The access to the region is complicated and it has no infrastructure for scientific research. During 14 days, the butterflies were collected with entomological nets, traps and Ahrenholz's technique in different environments in the park and its surroundings. A total of 482 species were identified, none of them present in red lists of endangered species. It is expected a significantly greater number of species after the addition of new collections in other seasons, as the Jacknife 1 estimate does not reach its asymptote, or as compared to inventories in nearby areas that list nearly 1700 species after a greater sampling effort.
\end{abstract}

Keywords: amazonian forest, butterflies, inventory, protected area.

MIELKE, O.H.H., CARNEIRO, E. \& CASAGRANDE, M.M. Lepidopterofauna (Papilionoidea e Hesperioidea) do Parque Estadual do Chandless e arredores, Acre, Brasil. Biota Neotrop. 10(4): http://www.biotaneotropica. org.br/v10n4/pt/abstract?inventory+bn03210042010.

Resumo: Tendo em vista a ausência de inventários lepidopterológicos no Estado do Acre e sua escassez no bioma amazônico brasileiro, o presente estudo objetivou contribuir para a construção da lista de Hesperioidea e Papilionoidea presentes no Parque Estadual do Chandless, localizado em uma região de difícil acesso e sem infraestrutura para pesquisa científica. Durante 14 dias foram realizadas coletas com redes entomológicas, armadilhas e técnica de Ahrenholz em diferentes ambientes que caracterizam o parque e seus arredores. Foram identificadas ao total 482 espécies, nenhuma delas presente em listas vermelhas de espécies ameaçadas. É esperado um número significativamente maior de espécies após a adição de novas coletas em outras estações do ano, visto a estimativa Jacknife 1 não atingir sua assíntota, ou mesmo em comparação a inventários em áreas próximas que listam, após um intenso esforço amostral, até cerca de 1700 espécies.

Palavras-chave: floresta amazônica, borboletas, inventário, unidade de conservação. 


\section{Introdução}

A grande e exuberante biodiversidade concentrada nos trópicos tem sido alvo de admiração do naturalismo desde a descoberta das Américas, culminando com a visita de naturalistas de expressão histórica como Darwin, Wallace, Bates e Müller (Lewinsohn et al. 2005). Entretanto, o conhecimento acumulado até então desta diversidade, tão qual comparada à diversidade estimada no mundo, ainda sugere uma larga ignorância de sua magnitude (ver Endler 1982, Erwin 1982, 1991, May 1988, Stork 1988, Gaston 1991). Como agravante, as políticas públicas brasileiras, ligadas a um país de maior biodiversidade do mundo (McNelly et al. 1990), ainda incentivam a conversão de grandes porções de hábitats naturais em paisagens antrópicas, onde se imagina que como consequência, resultou na extinção de muitas espécies antes mesmo de serem descritas (Stork 1988, Brown Jr. \& Brown 1992, Casagrande et al. 1998, Dunn 2005).

Os estudos que tem por objetivo inventariar a fauna e a flora locais são as ferramentas mais efetivas para o suprimento dessas lacunas. Além de prestar auxílio para a descoberta de novas espécies, também são considerados hoje fundamentais para a implementação de unidades de conservação (Terborgh \& Winter 1983, Scott et al. 1987), visando a contextualização da importância da área ou para a caracterização de suas particularidades. Além disso, os inventários também são ótimas ferramentas para a determinação dos limites de distribuição das espécies, contribuindo para o conhecimento de endemismos e áreas prioritárias para conservação (Lewinsohn et al. 2005). Seus resultados podem apontar a presença de espécies ameaçadas de extinção (Brown Jr. \& Freitas 2000b, Carneiro et al. 2008a) ou mesmo redescobrir populações já consideradas extintas.

Embora Lepidoptera seja certamente o grupo de insetos melhor inventariado dentre as quatro ordens megadiversas de insetos (Gaston 1991), este grau de inventariamento é somente elevado no hemisfério norte, e mesmo assim, sua contribuição sistemática é pouco relevante frente a toda sistemática de Lepidoptera (Kristensen et al. 2007). No Brasil em especial, a escassez de estudos dessa natureza é agravada pela má distribuição destes, altamente correlacionada com a ocupação humana incluindo a residência de pesquisadores, e que acabam por gerar lacunas no conhecimento, representadas por enormes áreas ainda sem quaisquer registros de fauna (Carneiro et al. 2008b).

Além de atrair um imenso carisma devido à beleza exuberante de suas formas e cores, as borboletas são usualmente citadas com potencial uso como espécies-bandeira (DeVries \& Walla 2001), espécies guarda-chuva (New 1997), indicadores ambientais (Brown Jr. \& Freitas 2000a), por apresentarem alta sensibilidade à alterações ambientais, mesmo em curto prazo, ou caracterização da tipologia ambiental atual ou histórica de uma determinada região (Gutiérrez 1997).

Tendo em vista a ausência de informações sobre a lepidopterofauna do Acre, as grandes dificuldades de acesso e logística para produção científica na região e as recomendações para produção prioritária desse tipo de estudo no bioma amazônico (Carneiro et al. 2008b), este estudo objetiva listar as espécies de borboletas presentes no Parque Estadual do Chandless e seus arredores, e assim contribuir para a catalogação da lepidopterofauna do Brasil.

\section{Material e Métodos}

Entre os dias 3 e 16 de agosto de 2008 foram realizadas coletas diárias no interior do Parque Estadual do Chandless, Santa Rosa do Purús e seus arredores, na região Centro-Sul do Acre. Diferentes ambientes foram percorridos a fim de inventariar a maior diversidade possível de lepidópteros (Brown Jr. 1972), onde se destacam: floresta ombrófila aberta, floresta ombrófila aberta com predominância de bambus (tabocais), praias do Rio Purús e regiões antropizadas, como os arredores da cidade e estradas que levam ao parque.
Três diferentes metodologias foram empregadas para coletas de grupos distintos de borboletas. A busca ativa de exemplares, método tradicionalmente utilizado para coleta de grande parte das espécies de Lepidoptera, deu-se mediante o uso de rede entomológica, onde foram percorridas trilhas pelas referidas localidades, entre o período de 9:00 às 16:00 horas. Adicionalmente, 10 armadilhas para Lepidoptera (padrão DeVries 1987), com iscas de peixe em decomposição como atrativo, foram penduradas a aproximadamente $1 \mathrm{~m}$ do solo no sub-bosque da floresta e da floresta com bambús. As armadilhas eram vistoriadas constantemente para retirada dos exemplares. Por fim, ao longo de cada trilha, foram depositados sobre a superfície adaxial de folhas, inúmeros fragmentos de guardanapos brancos umedecidos com saliva humana, utilizados como atrativo principal para espécies de Hesperiidae (técnica de Ahrenholz), como proposto por Lamas et al. (1993).

Todos os espécimes capturados foram sacrificados através de aperto no tórax, metodologia tradicionalmente utilizada para evitar a danificação das asas e necessária para identificação precisa a nível específico. Os procedimentos de coleta e preparação do material se mantiveram de acordo com as normas internacionais, enquanto as identificações se basearam em revisões e descrições de gêneros e espécies em literatura, e comparações com exemplares previamente identificados e disponíveis na Coleção de Entomologia Padre Jesus Santiago Moure, Departamento de Zoologia na Universidade Federal do Paraná. Nessa mesma coleção encontram-se depositados os exemplares capturados durante a expedição. A classificação sistemática adotada segue basicamente aquela proposta por Lamas (2004).

\section{Caracterização da área}

Localizado no Centro-Sul do Estado do Acre (Figura 1) e marginando a fronteira brasileira com o Perú, o Parque Estadual do Chandless é a segunda maior unidade de conservação do estado com 695,3 mil ha. A vegetação amazônica predominante é denominada Floresta Ombrófila Aberta, considerada uma forma de transição entre a floresta amazônica e áreas extra-amazônicas. Caracterizada por uma fisionomia florestal aberta, com árvores de baixa altura, raramente excedendo $20 \mathrm{~m}$, sub-bosque de três tipos característicos com predominância de bambus, palmeiras e cipós. O relevo é ondulado e varia entre 200-390 m de altitude. O clima da região é Ami quente e úmido, segundo a classificação de Köppen, e se caracteriza por uma pluviosidade relativamente baixa e alta sazonalidade, indicando variações pluviométricas entre $1566-2425 \mathrm{~mm}$. com precipitação média anual de $2.152 \mathrm{~mm}$ e período seco se estendendo a quatro meses no ano (Daly \& Silveira 2008).

\section{Resultados}

No Parque Estadual do Chandless e arredores foram confirmadas 482 espécies de borboletas, conforme Tabela 1, perfazendo a primeira lista de lepidopterofauna já publicada para uma localidade no Acre.

Sobre a composição de espécies encontradas, verificou-se Nymphalidae e Hesperiidae como as famílias mais ricas (172 e 168 spp. respectivamente), seguidos por Riodinidae (83 spp.), Lycaenidae (30 spp.), Pieridae (19 spp.) e Papilionidae (10 spp.) (Figura 2). Com relação às subfamílias, Pyrginae, Riodininae e Hesperiinae foram as mais representativas compreendendo cerca de metade de todas as espécies listadas (Figura 3).

\section{Discussão}

O número de espécies registrados no Parque Estadual do Chandless é consideravelmente baixo (apenas 482) quando comparado a inventários realizados em regiões próximas: Parque Nacional del Manu, Peru, 1300 spp. (Robbins et al. 1996); Tambopata, 


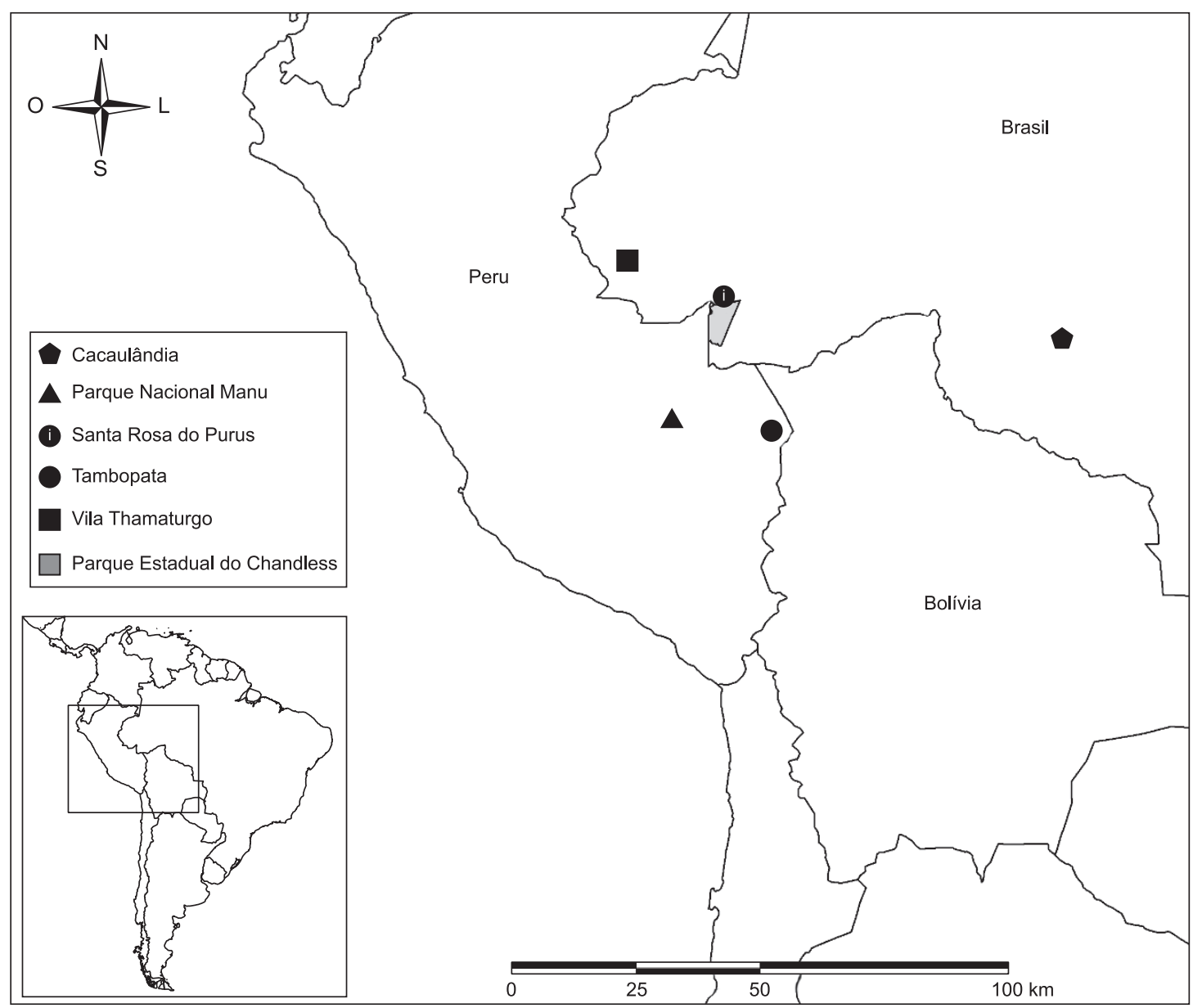

Figura 1. Mapa da localização do Parque Estadual do Chandless e inventários realizados em proximidades amazônicas.

Figure 1. Map from Parque Estadual do Chandless location with inventories made on amazon nearby localities.

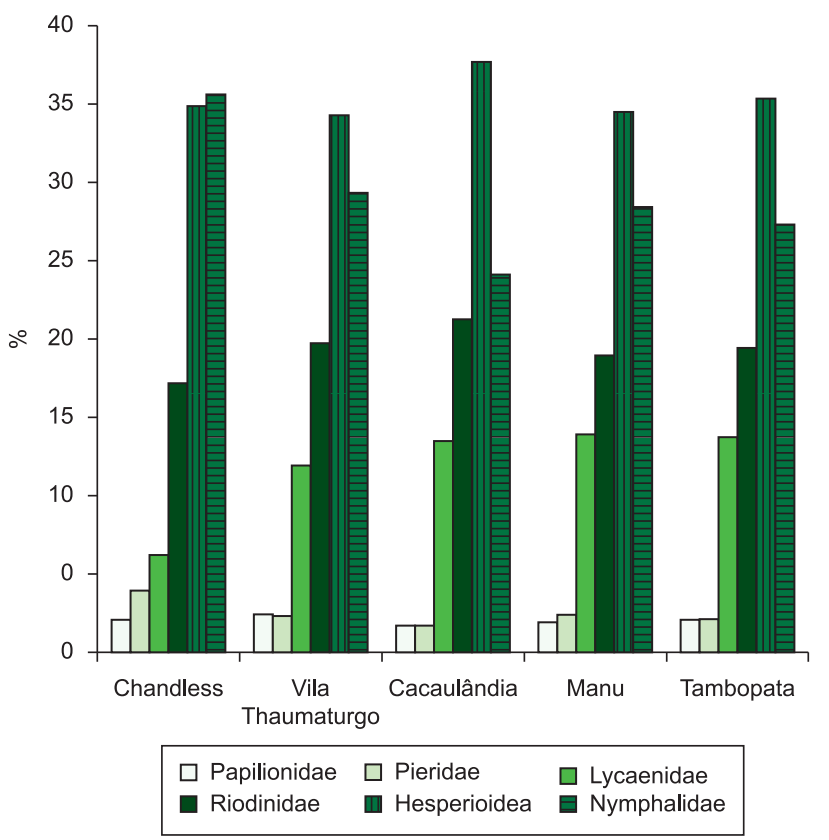

Figura 2. Percentual da riqueza entre as famílias de borboletas (Papilionoidea e Hesperioidea) do Parque Estadual do Chandless, comparada a inventários realizados em localidades próximas (Brown Jr. \& Freitas 2002).

Figure 2. Richness percentual distributed between butterfly families (Papilionoidea e Hesperioidea) from Parque Estadual do Chandless, compared to inventories realized on nearby localities (Brown Jr. \& Freitas 2002).

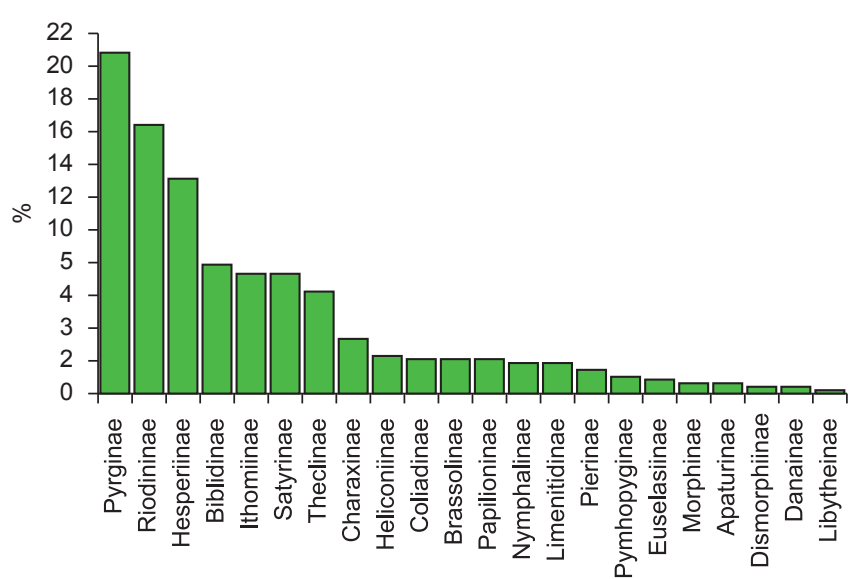

Figura 3. Percentual da riqueza entre as subfamílias de borboletas (Papilionoidea e Hesperioidea) do Parque Estadual do Chandless.

Figure 3. Richness percentual distributed between butterfly subfamilies (Papilionoidea e Hesperioidea) from Parque Estadual do Chandless. 
Mielke, O.H.H. et al.

Tabela 1. Lista de espécies de borboletas (Papilionoidea e Hesperioidea) encontradas no Parque Estadual do Chandless e arredores.

Table 1. Butterfly species list (Papilionoidea e Hesperioidea) registered in Parque Estadual do Chandless and surroundings.

\begin{tabular}{|c|c|}
\hline Papilionoidea & \\
\hline \multicolumn{2}{|l|}{ Papilionidae } \\
\hline \multicolumn{2}{|l|}{ Papilioninae } \\
\hline \multirow[t]{3}{*}{ Leptocircini } & Protesilaus protesilaus protesilaus (Linnaeus, 1758) \\
\hline & Protesilaus telesilaus telesilaus (Felder \& Felder, 1864) \\
\hline & Protographium agesilaus autosilaus (Bates, 1861) \\
\hline \multirow[t]{3}{*}{ Troidini } & Battus polydamas polydamas (Linnaeus, 1758) \\
\hline & Parides anchises drucei (Butler 1874) \\
\hline & Parides sesostris sesostris (Cramer, 1779) \\
\hline \multirow[t]{4}{*}{ Papilionini } & Heraclides anchisiades anchisiades (Esper, 1788) \\
\hline & Heraclides thoas cyniras (Ménétriés, 1857) \\
\hline & Heraclides torquatus torquatus (Cramer, 1777) \\
\hline & Pterourus zagreus nigroapicalis Bollino \& Sala, 1998 \\
\hline \multicolumn{2}{|l|}{ Pieridae } \\
\hline \multirow[t]{2}{*}{ Dismorphiinae } & Enantia lina galanthis (Bates, 1861) \\
\hline & Anteos menippe (Hübner, [1818]) \\
\hline \multirow[t]{10}{*}{ Coliadinae } & Aphrissa statira statira (Cramer, 1777) \\
\hline & Eurema albula albula (Cramer, 1775) \\
\hline & Eurema arbela arbela Geyer, 1832 \\
\hline & Eurema elathea obsoleta (Jörgensen, 1932) \\
\hline & Leucidia brephos (Hübner, [1809]) \\
\hline & Phoebis argante argante (Fabricius, 1775) \\
\hline & Phoebis philea philea (Linnaeus, 1763) \\
\hline & Phoenis sennae marcellina (Cramer, 1777) \\
\hline & Pyrisitia leuce leuce (Boisduval, 1836) \\
\hline & Rhabdodryas trite trite (Linnaeus, 1758) \\
\hline \multirow[t]{7}{*}{ Pierinae } & Ascia monusta automate (Burmeister, 1878) \\
\hline & Ganyra phaloe phaloe (Godart, 1819) \\
\hline & Glutophrissa drusilla drusilla (Cramer, 1777) \\
\hline & Itaballia demophile lucania (Fruhstorfer, 1907) \\
\hline & Itaballia pandosia pisonis ((Hewitson, 1861) \\
\hline & Perrhybris pamela mazuka Lamas, 1981 \\
\hline & Pieriballia viardi rubecula (Fruhstorfer, 1907) \\
\hline \multicolumn{2}{|l|}{ Nymphalidae } \\
\hline \multicolumn{2}{|l|}{ Danainae } \\
\hline \multirow[t]{2}{*}{ Euploeini } & Lycorea halia pales Felder \& Felder, 1862 \\
\hline & Danaus plexippus megalippe (Hübner, [1826]) \\
\hline \multicolumn{2}{|l|}{ Ithomiinae } \\
\hline \multirow[t]{2}{*}{ Tithoreini } & Tithorea harmonia brunnea Haensch, 1905 \\
\hline & Aeria eurimedia nigricola (Felder \& Felder, 1862) \\
\hline \multirow[t]{2}{*}{ Melinaeini } & Melinaea menophilus orestes Salvin, 1871 \\
\hline & Melinaea satevis lamasi K. Brown, 1977 \\
\hline \multirow[t]{7}{*}{ Mechanitini } & Methona confusa psamathe Godman \& Salvin, 1898 \\
\hline & Thyridia psidii ino Felder \& Felder, 1862 \\
\hline & Scada reckia labyrintha Lamas, 1985 \\
\hline & Forbestra olivencia aeneola Fox, 1967 \\
\hline & Mechanitis lysimnia menecles Hewitson, 1860 \\
\hline & Mechanitis mazaeus mazaeus Hewitson, 1860 \\
\hline & Mechanitis polymnia angustifascia Talbot, 1928 \\
\hline Napeogenini & Hypothyris cantobrica nundina (D'Almeida, 1945) \\
\hline
\end{tabular}


Tabela 1. Continuação...

Papilionoidea

Oleriini

Dircennini

Godyridini

Morphinae

Morphini

Brassolinae

Biini

Brassolini

Naropini

Satyrinae

Haeterini

Satyrini
Hypothyris euclea callanga (Haensch, 1905)

Hypothyris semifulva virgilini (Riley, 1919)

Napeogenes duessa duessa (Hewitson, [1859])

Napeogenes larina deucalion Haensch, 1905

Napeogenes pharo pharo (Felder \& Felder, 1862)

Napeogenes sylphis sylphis (Guérin-Méneville, [1844])

Hyposcada anchiala anchiala (Hewitson, 1868)

Oleria alexina didymaea (Hewitson, 1876)

Oleria astraea burchelli (Sanders, 1904)

Oleria gunilla ssp.

Oleria victorine graziella (Oberthür, 1879)

Ceratinia tutia fuscens (Haensch, 1905)

Callithomia alexirrhoe thornax Bates, 1862

Callithomia lenea zelie (Guérin-Méneville, [1844])

Dircenna loreta acreana D’Almeida, 1950

Pteronymia sao guntheri Lamas, 1985

Pteronymia vestilla ucaya Haensch, 1909

Hypoleria lavinia cajona Haensch, 1905

Brevioleria aelia brevicula (D'Almeida, 1951)

Brevioleria arzalia ssp.

Heterosais giulia nephele (Bates, 1862)

Pseudoscada florula ssp.

Pseudoscada timna ssp.

Antirrhea taygetina taygetina (Butler, 1868)

Morpho achilles phokylides Fruhstorfer, 1912

Morpho helenor theodorus Fruhstorfer, 1907

Bia actorion actorion (Linnaeus, 1763)

Caligo eurilochus pallidus Fruhstorfer, 1912

Caligo idomeneus idomenides Fruhstorfer, 1903

Caligo illioneus praxsiodus Fruhstorfer, 1912

Catoblepia berecynthia adjecta Stichel, 1906

Opotera aorsa hilara Stichel, 1902

Opsiphanes cassiae cassiae (Linnaeus, 1758)

Opsiphanes invirae amplificatus Stichel, 1904

Narope cyllabarus Westwood, 1851

Narope nesope Hewitson, 1869

Haetera piera pakitza Lamas, 1998.

Pierella astyoche stollei Ribeiro, 1931

Pierella hortona albofasciata Rosenberg \& Talbot, 1914

Pierella lamia chalybaea Godman, 1905

Pierella lena brasiliensis (Felder \& Felder, 1862)

Caeruleuptychia aegrota (Butler, 1867)

Caeruleuptychia caerulea (Butler, 1869)

Caeruleuptychia glauca (Weymer, 1911)

Caeruleuptychia helios (Weymer, 1911)

Chloreuptychia herseis (Godart, [1824])

Chloreuptychia tolumnia (Cramer, 1777)

Cissia penelope (Fabricius, 1775) 
Tabela 1. Continuação...

\section{Papilionoidea}

Charaxinae Anaeini

Preponini

Apaturinae

Biblidinae

Cyrestini

Biblidini
Cissia proba (Weymer, 1911)

Euptychia enyo Butler, 1867

Euptychia westwoodi Butler 1867

Harjesia oreba (Butler, 1870)

Hermeuptychia hermes (Fabricius, 1775)

Magneuptychia fugitiva Lamas, [1997]

Magneuptychia moderata (Weymer, 1911)

Magneuptychia ocypete (Fabricius, 1776)

Pareuptychia hesionides Forster, 1964

Posttaygetis penelea (Cramer, 1777)

Pseudodebis marpessa (Hewitson, 1862)

Rareuptychia clio (Weymer, 1911)

Splendeuptychia ashna (Hewitson, 1869)

Splendeuptychia aurigera (Weymer, [1811])

Taygetis cleopatra Felder \& Felder, 1867

Taygetis rufomarginata Staudinger, 1888

Taygetis sylvia Bates, 1866

Taygetomorpha celia (Cramer, 1779)

Yphthymoides argyrospila (Butler, 1867)

Yphthimoides sp.

Zischkaia amalda (Weymer, 1911)

Gen. sp. ign.

Amphidecta calliomma (Felder \& Felder, 1862)

Consul fabius fulvus (Butler, 1875)

Fountainea halice halice (Godart, [1824])

Fountainea ryphea ryphea (Cramer, 1775)

Memphis acidalia memphis (Felder \& Felder, 1867)

Memphis glauce glauce (Felder \& Felder, 1862)

Memphis lemnos (H. Druce, 1877)

Memphis moruus morpheus (Staudinger, [1886])

Memphis phantes phantes (Hopffer, 1874)

Memphis polycarmes (Fabricius, 1775)

Memphis praxias praxias (Hopffer, 1874)

Memphis xenocles xenocles (Westwood, 1850)

Zaretis itys itys (Cramer, 1777)

Agrias claudina sardanapalus Bates, 1860

Archaeoprepona demophon demophon (Linnaeus, 1758)

Prepona laertes demodice (Godart, [1824])

Prepona pylene eugenes Bates, 1865

Doxocopa agathina agathina (Cramer, 1777)

Doxocopa lavinia (Butler, 1866)

Doxocopa pavon pavon (Latreille, [1809])

Marpesia chiron marius (Cramer, 1779)

Marpesia egina (Bates, 1865)

Marpesia furcula oechalia (Westwood, 1850)

Marpesia petreus petreus (Cramer, 1776)

Marpesia themistocles norica (Hewitson, 1852)

Biblis hyperia laticlavia (Thieme, 1904)

Vila azeca azeca (Doubleday, [1848]) 
Tabela 1. Continuação...

\section{Papilionoidea}

Nymphalinae

Coeini

Kallimini

Melitaeini

Limenitidinae

Limenitidini
Vila emilia caecilia (Felder \& Felder, 1862)

Vila cacica Staudinger, 1886

Eunica clytia (Hewitson, 1852)

Eunica caelina alycia Fruhstorfer, 1909

Eunica eurota eurota (Cramer, 1775)

Eunica marsolia fasula Jenkins, 1990

Eunica mygdonia mygdonia (Godart, [1824])

Eunica tatila bellaria Frustorfer, 1908

Eunica volumna celma (Hewitson, 1852)

Myscelia capenas capena (Hewitson, [1857])

Nessaea hewitsonii boliviensis Jenkins, 1989

Nessaea obrinus lesoudieri Le Moult, 1933

Batesia hypochlora hypoxantha Salvin \& Godman, 1868

Hamadryas arinome arinome (Lucas, 1853)

Hamadryas chloe chloe (Stoll, 1782)

Panacaea prola amazonica Fruhstorfer, 1915

Epiphile lampethusa lampethusa Doubleday, [1848]

Nica flavilla sylvestris Bates, 1864

Pyrrhogyra amphiro amphiro Bates, 1865

Pyrrhogyra crameri hagnodorus Frustorfer, 1908

Pyrrhogyra otolais olivença Bates, 1864

Temenis laothoe laothoe (Cramer, 1777)

Dynamine aerata (Butler, 1877)

Dynamine agacles agacles (Dalman, 1823)

Dynamine athemon barreiroi Fernández, 1928

Dynamine artemisia glauce (Bates, 1865)

Dynamine chryseis (Bates, 1865)

Dynamine gisella (Hewitson, 1857)

Dynamine sosthenes smerdis Tessmann, 1928

Diaethria clymena peruviana (Guenée, 1872)

Paulogramma pyracmon peristera (Hewitson, 1853)

Baeotus deucalion (Felder \& Felder, 1860)

Historis acheronta acheronta (Fabricius, 1775)

Historis odius dious Lamas, 1995

Tigridia acesta tapajona (Butler, 1873)

Anartia jatrophae jatrophae (Linnaeus, 1763)

Junonia evarete evarete (Cramer, 1779)

Eresia clio clio (Linnaeus, 1758)

Eresia eunice eunice (Hübner, [1807])

Telenassa teletusa burchelli (Moulton, 1909)

Adelpha attica attica (Felder \& Felder, 1867)

Adelpha cocala cocala (Cramer, 1779)

Adelpha epione epione (Godart, [1824])

Adelpha iphiclus iphiclus (Linnaeus, 1758)

Adelpha jordani Fruhstorfer, 1913

Adelpha malea aethalia (Felder \& Felder, 1861)

Adelpha messana delphicola Fruhstorfer, 1910

Adelpha plesaure phliassa (Godart, [1824]

Actinote pellenea hyalina Jordan, 1913 
Tabela 1. Continuação...

Papilionoidea

Heliconiinae

Acraeini

Heliconiini

Libytheinae

Riodinidae

Euselasiinae

Euselasiini

Riodininae

Mesosemiini

Eurybiini

Riodinini
Dryas iulia alcionea (Cramer, 1779)

Philaethria dido dido (Linnaeus, 1763)

Neruda aeode manu (Lamas, 1976)

Eueides aliphera aliphera (Godart, 1819)

Eueides isabella huebneri Ménétriés, 1857

Heliconius burneyi koenigi Neukirchen, 1995

Heliconius erato luscombei Lamas, 1976

Heliconius melpomene schunkei Lamas, 1976

Heliconius numata lyrcaeus Weymer, 1891

Heliconius sara thamar (Hübner, 1806)

Heliconius wallacei flavescens Weymer, 1891

Libytheana carinenta carinenta (Cramer, 1777)

Euselasia arbas (Stoll, 1781)

Euselasia authe ocalea (H. Druce, 1904)

Euselasia eutychus (Hewitson, 1856)

Euselasia sp.

Mesosemia judicialis (Butler, 1874)

Mesosemia misipsa Hewitson 1859

Mesosemia sirenia (Stichel, 1909)

Mesosemia sp. 1

Mesosemia sp. 2

Semomesia croesus siccata Stichel, 1919

Ithomiola floralis celtina (Hewitson, 1870)

Ithomiola orpheus (Westwood, 1851)

Hyphilaria parthenis (Westwood, 1851)

Napaea beltiana (Bates, 1867)

Eurybia franciscana Felder \& Felder, 1862

Eurybia halimede halimede (Hübner, [1807])

Eurybia nicaeus nicaeus (Fabricius, 1775)

Alesa amesis (Cramer, 1777)

Alesa telephae (Godart, [1824])

Ancyluris melior Stichel, 1910

Rhetus arcius huana (Saunders, 1859)

Rhetus periander periander (Cramer, 1777)

Chalodeta theodora (Felder \& Felder, 1862)

Metacharis lucius (Fabricius, 1793)

Cartea vitula vitula (Hewitson, [1853])

Cartea ucayala Thieme, 1907

Charis anius (Cramer, 1776)

Detritivora cleonus (Stoll, 1781)

Detritivora zama (Bates, 1868)

Caria plutargus amazonica (Bates, 1868)

Caria sp. 1

Caria sp. 2

Caria sp. 3

Crocozona coecias coecias (Hewitson, 1866)

Lasaia agesilas agesilas (Latreille, [1809]) 
Tabela 1. Continuação...

Papilionoidea

Symmachiini

Helicopini

inc. sedis

Nymphidiini

Stalachtini

Lycaenidae

Theclinae

Eumaeini
Amarynthis meneria (Cramer, 1776)

Riodina lysippus lysias Stichel, 1910

Melanis smithiae smithiae (Westwood, 1851)

Mesene leucophrys Bates, 1868

Mesene sp.

Sarota acantus (Stoll, 1782)

Sarota completa Hall, 1998

Anteros formosus formosus (Cramer, 1777)

Ourocnemis archytas (Stoll, 1787)

Calydna caieta Hewitson, 1854

Calydna catana Hewitson, 1859

Calydna cea Hewitson, 1859

Calydna charila Hewitson, 1854

Calydna hiria (Godart, [1824])

Calydna micra Bates, 1868

Emesis condigna Stichel, 1925

Emesis mandana mandana (Cramer, 1780)

Emesis ocypore ocypore (Geyer, 1837)

Emesis temesa temesa (Hewitson, 1870)

Emesis spreta Bates, 1868

Thisbe hyalina (Butler, 1867)

Thisbe irenea ssp.

Juditha azan completa (Lathy, 1904)

Juditha sp. 1

Synargis orestessa Hübner, [1819]

Menander pretus pretus (Cramer, 1777)

Calospila parthaon (Dalman, 1823)

Calospila rhesa (Hewitson, 1858)

Calospila sp.

Adelotypa epixanthe (Stichel, 1911)

Adelotypa leucophaea (Hübner, [1821])

Adelotypa tinea (Bates, 1868)

Adelotypa violacea (Butler, 1867)

Setabis epitus epiphanis (Stichel, 1910)

Setabis flammula (Bates, 1868)

Nymphidium acherois erymanthus Ménétriés, 1855

Nymphidium ascolia augea $\mathrm{H}$. Druce, 1904

Nymphidium azanoides amazonensis Callaghan, 1986

Nymphidium baeotia Hewitson, [1853]

Nymphidium caricae parthenium Stichel, 1924

Nymphidium lisimon lisimon (Stoll, 1790)

Nymphidium leucosa medusa H. Druce, 1904

Nymphidium omois Hewitson, 1865

Theope eurygonina Bates, 1868

Theope nycteis (Westwood, 1851)

Theope pedias Herrich-Schäffer, [1853]

Stalachtis calliope calliope (Linnaeus, 1758)

Stalachtis euterpe latefasciata Staudinger, 1888 
Tabela 1. Continuação...

\begin{tabular}{|c|c|}
\hline Papilionoidea & \\
\hline & Arawacus separata (Lathy, 1926) \\
\hline & Calycopis malta (Schaus, 1902) \\
\hline & Calycopis trebula (Hewitson, 1868) \\
\hline & Calycopis orcillula (Strand, 1916) \\
\hline & Calycopis bellera (Hewitson, 1877) \\
\hline & Calycopis cerata (Hewitson, 1877) \\
\hline & Calycopis sp. \\
\hline & Chalybs janias (Cramer, 1779) \\
\hline & Chlorostrymon telea (Hewitson, 1868) \\
\hline & Celmia celmus (Cramer, 1775) \\
\hline & Dicya carnica (Hewitson, 1873) \\
\hline & Eumaeus toxana (Boisduval, 1870) \\
\hline & Exorbaeta metanira (Hewitson, 1867) \\
\hline & Janthecla leea Venables \& Robbins, 1991 \\
\hline & Janthecla sista (Hewitson, 1867) \\
\hline & Kolana ligurina (Hewitson, 1874) \\
\hline & Ocaria ocrisia (Hewitson, 1868) \\
\hline & Paiwarria telemus (Cramer, 1775) \\
\hline & Panthiades phaleros (Linnaeus, 1767) \\
\hline & Siderus athymbra (Hewitson, 1867) \\
\hline & Siderus leucophaeus (Hübner, [1813]) \\
\hline & Strephonota purpurantes (H. H. Druce, 1907) \\
\hline & Strephonota sphinx (Fabricius, 1775) \\
\hline & Strymon sp. \\
\hline & Theritas hemon (Cramer, 1775) \\
\hline & Tmolus cydrara (Hewitson, 1868) \\
\hline & Tmolus echion (Linnaeus, 1767) \\
\hline & Strephonota cylarissus (Herbst, 1800) \\
\hline & Ziegleria hesperitis (Butler \& H. Druce, 1872) \\
\hline \multicolumn{2}{|l|}{ Hesperioidea } \\
\hline \multicolumn{2}{|l|}{ Hesperiidae } \\
\hline \multicolumn{2}{|l|}{ Pyrrhopyginae } \\
\hline \multirow[t]{2}{*}{ Passovini } & Myscelus amystis mysus Evans, 1951 \\
\hline & Myscelus epimachia epimachia Herrich-Schäffer, 1869 \\
\hline \multirow[t]{3}{*}{ Pyrrhopygini } & Elbella etna Evans, 1951 \\
\hline & Elbella intersecta intersecta (Herrich-Schäffer, 1869) \\
\hline & Jemadia gnetus (Fabricius, 1781) \\
\hline \multicolumn{2}{|l|}{ Pyrginae } \\
\hline \multirow[t]{13}{*}{ Eudamini } & Aguna coelus (Stoll, 1781) \\
\hline & Aguna coeloides Austin \& Mielke, 1998 \\
\hline & Aguna latifascia Austin \& Mielke, 1998 \\
\hline & Aguna metophis (Latreille, [1824]) \\
\hline & Aguna parva Austin \& Mielke, 1998 \\
\hline & Aguna squamalba Austin \& Mielke, 1998 \\
\hline & Aguna sp. \\
\hline & Astraptes alardus alardus (Stoll, 1790) \\
\hline & Astraptes alector hoppferi (Plötz, 1881) \\
\hline & Astraptes cretatus cretatus (Hayward, 1939) \\
\hline & Astraptes creteus creteus (Cramer, 1780) \\
\hline & Astraptes fulgerator fulgerator (Walch, 1775) \\
\hline & Astraptes latimargo tinda Evans, 1952 \\
\hline
\end{tabular}


Tabela 1. Continuação...

\section{Hesperioidea}

Pyrgini

Augiades crinisus (Cramer, 1780)

Bungalotis midas (Cramer, 1775)

Cabirus procas junta Evans, 1952

Celaenorrhinus jao (Mabille, 1889)

Celaenorrhinus sp.

Chrysoplectrum bahiana bahiana (Herrich-Schäffer, 1869)

Codatractus sp.

Drephalys (Drephalys) alcmon (Cramer, 1779)

Entheus matho dius Mabille, 1898

Entheus latebrosus Austin, 1997

Epargyreus clavicornis clavicornis (Herrich-Schäffer, 1869)

Hyalothyrus neleus neleus (Linnaeus, 1758)

Phanus ecitonorum Austin, 1993

Phanus marshalli (Kirby, 1880)

Phanus vitreus (Stoll, 1781)

Polythrix auginus (Hewitson, 1867)

Polythrix octomaculata (Sepp, [1844])

Proteides mercurius mercurius (Fabricius, 1787)

Urbanus chalco (Hübner, 1823)

Urbanus dorantes dorantes (Stoll, 1790)

Urbanus doryssus doryssus (Swainson, 1831)

Urbanus esma Evans, 1952

Urbanus reductus (Riley, 1919)

Urbanus simplicius (Stoll, 1790)

Urbanus teleus (Hübner, 1821)

Urbanus velinus (Plötz, 1880)

Achlyodes busirus heros Ehrmann, 1909

Achlyodes mithridates thraso (Hübner, [1807])

Aethilla echina echina Hewitsoin, 1870

Anastrus neaeris narva Evans, 1953

Anastrus sempiternus simplicior (Möschler, 1877)

Anastrus tolimus robigus (Plötz, 1884)

Anisochoria pedaliodina pedaliodina (Butler, 1870)

Antigonus erosus (Hübner, [1812])

Antigonus decens Butler, 1874

Antigonus nearchus (Latreille, 1817)

Bolla atahuallpai (Lindsey, 1925)

Bolla cupreiceps (Mabille, 1891)

Carrhenes leada (Butler, 1870)

Carrhenes fuscescens conia Evans, 1953

Cycloglypha enega (Möschler, 1877)

Cyclosemia elelea (Hewitson 1878)

Cyclosemia herennius herennius (Stoll, 1782)

Ebrietas anacreon anacreon (Staudinger, 1876)

Ebrietas evanidus Mabille, 1898

Eracon paulinus (Stoll, 1782)

Gorgopas chlorocephala chlorocephala (Herrich-Schäffer, 1870)

Gorgopas trochilus (Hopffer, 1874)

Gorgythion beggina escalophoides Evans, 1953

Gorgythion plautia (Möschler, 1877) 
Tabela 1. Continuação...

\section{Hesperioidea}

Hesperiinae

Helias phalaenoides phalaenoides Fabricius, 1807

Heliopetes alana (Reakirt, 1868)

Marela tamyris tamba Evans, 1953

Milanion pilumnus pilumnus Mabille \& Boullet, 1917

Morvina fissimacula rema Evans, 1953

Myrinia santa santa Evans, 1953

Nisoniades macarius (Herrich-Schäffer, 1870)

Nisoniades mimas (Cramer, 1775)

Nisoniades rimana (Bell, 1942)

Ouleus accedens noctis (Lindsey, 1925)

Ouleus fridericus fridericus (Geyer, 1832)

Ouleus juxta juxta (Bell, 1934)

Paches trifasciatus Lindsey, 1925

Pachyuneuria herophile (Hayward, 1940)

Pellicia klugi Williams \& Bell, 1939

Plumbago plumbago (Plötz, 1884)

Polyctor polyctor polyctor (Prittwitz, 1868)

Pyrgus orcus (Stoll, 1780)

Pythonides grandis assecla Mabille, 1883

Pythonides herennius herennius (Geyer, 1832)

Pythonides maraca Mielke \& Casagrande, 1992

Quadrus cerialis (Stoll, 1782)

Quadrus contubernalis contubernalis (Mabille, 1883)

Quadrus deyrollei porta Evans, 1953

Sostrata pusilla pusilla Godman \& Salvin, 1895

Spioniades artemides (Stoll, 1782)

Staphylus astra (Williams \& Bell, 1940)

Staphylus lizeri lizeri (Hayward, 1938)

Staphylus melangon melangon (Mabille, 1883)

Staphylus oeta (Plötz, 1884)

Staphylus putumayo (Bell, 1937)

Telemiades amphion misitheus Mabille, 1888

Telemiades antiope tosca Evans, 1953

Telemiades delalande (Latreille, [1824])

Telemiades penidas (Hewitson, 1867)

Telemiades sp.

Zera tetrastigma tetrastigma (Sepp, [1847])

Aides duma argyrina Cowan, 1970

Alera manu Mielke \& Casagrande, 2004

Arita arita (Schaus, 1902)

Artines focus Evans, 1955

Callimormus corades (Felder, 1862)

Callimormus radiola radiola (Mabille, 1878)

Carystus hocus Evans, 1955

Carystus ploetzi Mielke \& Casagrande, 2002

Conga chydaea (Butler, 1877)

Cymaenes alumna (Butler, 1877)

Cymaenes sp.

Decinea decinea derisor (Mabille, 1891)

Ebusus ebusus ebusus (Cramer, 1780)

Enosis blotta Evans, 1955 
Tabela 1. Continuação...

Flaccilla aecas (Stoll, 1781)

Joanna boxi Evans, 1955

Lycas godart godart (Latreille, [1824])

Moeros moeros (Möschler, 1877)

Molo sp.

Morys geisa geisa (Möschler, 1879)

Eutychide subpunctata intermedia de Jong, 1983

Neoxeniades braesia braesia (Hewitson, 1867)

Nyctelius nyctelius nyctelius (Latreille, [1824])

Onophas columbaria flossites (M. R. Butler, 1874)

Orthos orthos orthos (Godman, 1900)

Oxynthes corusca (Herrich-Schäffer, 1869)

Parphorus decorus (Herrich-Schäffer, 1869)

Parphorus storax storax (Mabille, 1891)

Parphorus sp. 1

Parphorus sp. 2

Penicula advena advena (Draudt, 1923)

Perichares philetes philetes (Gmelin, [1790])

Pompeius pompeius (Latreille, [1824])

Propapias sipariana (Kaye, 1925)

Quasimellana angra Evans, 1955

Racta apella raza Evans, 1955

Remella remus (Fabricius, 1798)

Saliana fusta Evans, 1955

Saliana saladin culta Evans, 1955

Saliana longirostris (Sepp, [1840])

Saliana triangularis (Kaye, 1914)

Saturnus metonidia (Schaus, 1902)

Saturnus reticulata suffuscus (Hayward, 1939)

Synapte sp.

Thoon ranka Evans, 1955

Thoon sp.

Thracides cleanthes telmela (Hewitson, 1866)

Thracides panimeron H. H. Druce, 1908

Tisias quadrata quadrata (Herrich-Schäffer, 1869)

Tromba tromba Evans, 1955

Vehilius stictomenes stictomenes (Butler, 1877)

Vehilius vetula (Mabille, 1878)

Vehilius sp.1

Vehilius sp.2

Venas caerulans (Mabille, 1878)

Venas evans (Butler, 1877)

Vettius marcus marcus (Fabricius, 1787)

Vettius monacha (Plötz, 1882)

Vettius phyllus phyllus (Cramer, 1777)

Pharaeus sp.

Vinius tryhana tryhana (Kaye, 1914)

Xeniades orchamus orchamus (Cramer, 1777) 
Peru, 1234 spp. (Lamas 1981, 1985); Vila Thaumaturgo, Acre, 1620 spp. (Brown Jr. \& Freitas 2002); e Cacaulândia, Rondonia, 1730 spp. (Brown Jr. \& Freitas 2002). Todavia, quando comparado com inventário realizado com esforço amostral comparável ou similar, já se mostra semelhante: Ilha de Maracá, Roraima (Mielke \& Casagrande 1991).

A composição faunística é igualmente afetada pela discrepância amostral entre os levantamentos. Famílias como Hesperiidae, Lycaenidae e Riodinidae, as quais oferecem maiores dificuldades de amostragem, são normalmente subestimadas em estudos com duração inferior a um ou dois anos. Desta forma, Pieridae, Papilionidae e Nymphalidae são as famílias a serem melhor representadas neste tipo de estudo, a última superando significativamente os 25 a $29 \%$ do sugerido por Brown \& Freitas (1999) para quaisquer comunidades neotropicais. A predodimnância de Pyrginae, Riodininae e Hesperiinae é um fator já relatado por Ebert (1969), por se tratarem de espécies de pequeno tamanho e baixa frequência. Segundo o autor, tais grupos juntos com Theclinae corresponderiam a cerca de um terço de um total de uma comunidade neotropical.

A ausência de espécies ameaçadas de extinção na região sugere que a mesma ainda esteja em bom estado de conservação, embora não haja quaisquer estudos representativos para o Estado do Acre. Na lista brasileira de espécies ameaçadas somente há uma espécie que ocorre no Acre: Paititia neglecta Lamas, 1979 (Nymphalidae, Ithomiinae). Considerando que a espécie é muito mal conhecida e que também não houve um trabalho de campo considerável para sua indicação, a espécie talvez não devesse constar na lista.

O levantamento mostrou uma composição da fauna lepidopterológica característica para o Acre e Leste do Peru, assim como do Oeste de Rondônia, pois em sua maioria compartilham das mesmas espécies ou mesmo subespécies. Não foi possível compará-lo com outras regiões amazônicas próximas, devido à falta de inventários publicados. Assim o sendo, julgamos que é de extrema necessidade realizar levantamentos mais demorados (aproximadamente 5 anos) e consequentemente detalhados, afim de poder realizar comparações mais bem elaboradas.

\section{Agradecimentos}

À organização não governamental SOS Amazônia pela coordenação geral do plano de manejo em nome de sua diretora geral Silvia Helena Costa Brilhante e de seu coordenador científico Roberto Antonelli Filho. A Pierre Jauffret, Santo Antonio de Tauá, Pará, pela identificação de parte dos exemplares de Riodinidae.

\section{Referências Bibliográficas}

BROSE, U. \& MARTINEZ, N.D. 2004. Estimating the richness of species with variable mobility. Oikos 105:292-300.

BROWN Jr., K.S. 1972. Maximizing daily butterfly counts. J. Lepid. Soc. 26(3):183-196.

BROWN Jr., K.S. \& BROWN, G.G. 1992. Habitat alteration and species loss in Brazilian forests. In Tropical Deforestation and Species Extinction (T.C. Whitmore \& Sayer J.A., ed.). Chapman \& Hall, Londres, p.119-142.

BROWN, J.K.S. \& FREITAS, A.V.L. 1999. Lepidoptera. In Biodiversidade do Estado de São Paulo, Brasil: síntese do conhecimento ao final do século XX, invertebrados terrestres ( C.R.F. Brandão \& E.M. Cancello). USP, São Paulo, p.225-243.

BROWN Jr., K.S. \& FREITAS, A.V.L. 2000a. Atlantic Forest Butterflies: indicators for landscape consevation. Biotropica 32(4b):934-956.

BROWN Jr., K.S. \& FREITAS, A.V.L. 2000b. Diversidade de Lepidoptera em Santa Teresa, Espírito Santo. Bol. Mus. Biol. Mello Leitão (n. ser.) 11/12:71-118.
BROWN Jr., K.S. \& FREITAS, A.V.L. 2002. Diversidade Biológica no Alto Juruá: avaliação, causas e manutenção. In Enciclopedia da Floresta - O Alto Juruá: práticas e conhecimento das populações (M.M.C. Cunha \& M.B. Almeida, org.). Companhia das Letras, São Paulo, p.33-42.

CARNEIRO, E., MIELKE, O.H.H. \& CASAGRANDE, M.M. 2008 a. Borboletas do sul da ilha da Santa Catarina, Florianópolis, Santa Catarina, Brasil (Lepidoptera: Hesperioidea e Papilionoidea). SHILAP, Rev. Lepidopt. 36:261-271.

CARNEIRO, E., MIELKE, O.H.H. \& CASAGRANDE, M.M. 2008b. Inventários de borboletas no Brasil: estado da arte e modelo de áreas prioritárias para pesquisa com vistas à conservação. Nat. Conservação 6(2):68-90.

CASAGRANDE, M.M., MIELKE, O.H.H. \& BROWN Jr., K.S. 1998. Borboletas (Lepidoptera) ameaçadas de extinção em Minas Gerais, Brasil. Rev. Bras. Zool. 15(1):241-259.

COLWELL, R.K. 1997. EstimateS: Statistical estimation of species richness and share species from samples. User's Guide and application. http:// viceoy.eeb.uconn.edu?estimates (último acesso em: 20/10/2009).

DALY, D.C. \& SILVEIRA, M. 2008. Flora do Acre, Brasil. EDUFAC, Rio Branco, 555p.

DEVRIES, P.J. 1987. The Butterflies of Costa Rica and their natural history: Papilionidae, Pieridae, and Nymphalidae. Princeton Univ. Press., New Jersey, 327p.

DEVRIES, P.J. \& WALLA, T.R. 2001. Species diversity and community structure in neotropical fruit-feeding butterflies. Biol. J. Linn. Soc. 74:1-15.

DUNN, R.R. 2005. Modern insect extinctions, the neglected majority. Conserv. Biol. 19(4):1030-1036.

EBERT, H. 1969. On the frequency of butterflies in eastern Brazil, with a list of the butterfly fauna of Poços de Caldas, Minas Gerais. J. Lepid. Soc. 23(Suppl. 3):1-48.

ENDLER, J. 1982. Problems in distinguishing historical from ecological factors in biogeography. Am. Zool. 22:441-452.

ERWIN, T.L. 1982. Tropical forests: their richness in Coleoptera and other arthropod species. Coleopts. Bull. 36:74-75.

ERWIN, T.L. 1991. How many species are there? Revisited. Conserv. Biol. 5:1-4.

GASTON, K.J. 1991. The magnitude of global insect species richness. Conserv. Biol. 5:283-296.

GUTIÉRREZ, D. 1997. Importance of historical factors on species richness and composition of butterfly assemblages (Lepidoptera: Rhopalocera) in a northern Iberian mountain range. J. Biogeogr. 24:77-88.

KRISTENSEN, N.P., SCOBLE, M.J. \& KARSHOLT, O. 2007. Lepidoptera phylogeny and systematics: the state of inventorying moth and butterfly diversity. Zootaxa 1668:699-747.

LAMAS, G. 1981. La fauna de mariposas de la Reserva de Tambopata, Madre de Dios, Perú (Lepidoptera, Papilionoidea y Hesperioidea). Rev. Soc. Mex. Lepid. 6(2):23-40.

LAMAS, G. 1985. Los Papilionoidea (Lepidoptera) de la zona reservada de Tambopata, Madre de Dios, Perú. I. Papilionidae, Pieridae y Nymphalidae. Rev. Per. Ent. 27:59-73.

LAMAS, G., MIELKE, O.H.H. \& ROBBINS, R.K. 1993. The Ahrenholtz technique for attracting tropical skippers (Hesperiidae). J. Lepid. Soc. 47(1):80-82.

LAMAS, G. (ed.). 2004. Checklist: Part 4a. Hesperioidea - Papilionoidea. In Atlas of Neotropical Lepidoptera (J.B. Heppner, ed.). vol.5A. Association of Tropical Lepidoptera, Gainesville, 439p.

LEWINSOHN, T.M., FREITAS, A.V.L. \& PRADO, P.I. 2005. Conservação de Invertebrados terrestres e seus hábitats no brasil. Megadiversidade 1(1):62-69. 
MAY, R. 1988. How many species are there on Earth? Science 241:1441-1443.

McNELLY, J.A., MILLER, K.R., REID, W.V., MITTERMEIER, R.A. \& WERNER, T.B. 1990. Conserving the worlds biological diversity. IUCN, Gland; WRI/CI/WWF/World Bank, Washington, D.C., 193p.

MIELKE, O.H.H. \& CASAGRANDE, M.M. 1991. Lepidoptera: Papilionoidea e Hesperioidea coletados na Ilha de Maracá, Alto Alegre, Roraima, parte do projeto Maracá, com uma lista complementar de Hesperiidae de Roraima. Acta Amaz. 21:175-210.

NEW, T.R. 1997. Are Lepidoptera an effective "umbrella group" for biodiversity conservation? J. Insect. Conserv. 1(1):5-12.
ROBBINS, R.K., LAMAS, G., MIELKE, O.H.H., HARVEY, D.J. \& CASAGRANDE, M.M. 1996. Taxonomic composition and ecological structure of the species-rich butterfly community at Pakitza, Parque Nacional del Manu, Perú. In Manu. The Biodiversity of Southeastern Peru. La Biodiversidad del Sureste del Perú (D.E. Wilson \& Sandoval, A., ed.). Editorial Horizonte, Lima. p.217-252.

SCOTT, J.M., CSUTI, B., JACOBI, J.D. \& ESTES, J.E. 1987. A geographic approach to protecting future biological diversity. Bioscience 37:782-788.

STORK, N.E. 1988. Insect diversity: facts, fiction and speculation. Biol. J. Linn. Soc. 35:321-337.

TERBORGH, J. \& WINTER, B. 1983. A method for siting parks and reserves with special reference to Colombia and Ecuador. Biol. Conserv. 27:45-58. 
